\title{
Understanding local dwarf spheroidals and their scaling relations under MOdified Newtonian Dynamics
}

\author{
X. Hernandez ${ }^{1,2}$, S. Mendoza ${ }^{1}$, T. Suarez ${ }^{1}$, and T. Bernal ${ }^{1}$ \\ 1 Instituto de Astronomía, Universidad Nacional Autónoma de Mexico, AP 70-264, Ciudad Universitaria, \\ Distrito Federal 04510, Mexico \\ e-mail: [xavier; sergio; tsuarez; tbernal] @astroscu.unam.mx \\ 2 GEPI, Observatoire de Paris, Meudon Cedex, France
}

Received 15 September 2009 / Accepted 23 November 2009

\begin{abstract}
We use a specific form of the interpolation function in the MOND formalism, which optimally accounts for the internal structure of dwarf spheroidal (dSph) galaxies, to explore the consequences it has on the scaling relations seen in these systems. The particular form of the interpolation function we used leads to a law of gravity that does not degrade the good fit of the MOND proposal on galactic scales, and in fact, slightly improves the accordance with observations on dSph scales. This formalism yields a good description of gravitational phenomena without the need of invoking any still undetected and hypothetically dominant dark matter, in the weak field regime probed by local dSph galaxies. Isothermal equilibrium density profiles then yield projected surface density profiles for the local dSph galaxies in very good agreement with observational determinations, for values of the relevant parameters as inferred from recent observations of these Galactic satellites. The observed scaling relations for these systems are also naturally accounted for within the proposed scheme, including a previously unrecognised correlation of the inferred mass-to-light ratios of local dSph's with the ages of their stellar populations, which is natural in modified gravity schemes in the absence of dark matter. The results shed some light on the form that the MOND interpolating function may have in the most challenging regime, which occurs at moderate accelerations and intermediate mass-weighted lengths.
\end{abstract}

Key words. gravitation - galaxies: dwarf - galaxies: kinematics and dynamics - dark matter - Local Group

\section{Introduction}

If one wants to explore options where the existence of dark matter is not a necessity, one must consider variations in the law of gravity on galactic and extra-galactic scales where dark matter has been proposed to account for the observed dynamics. The best-studied such proposal is the MOdified Newtonian Dynamics (MOND) hypothesis of Milgrom (1983), which has been shown to account for the rotation curves of spiral galaxies (e.g. Sanders \& McGaugh 2002), naturally incorporating the Tully-Fisher relation, see for example Milgrom (2008a) and references therein.

Abundant recent publications of the velocity dispersion measurements of stars in the local dwarf spheroidal (dSph) galaxies, the extended and flat rotation curves of spiral galaxies, the high dispersion velocities of galaxies in clusters, the gravitational lensing due to massive clusters of galaxies, and even the cosmologically inferred matter content for the universe, are all successfully modelled using MOND, not as indirect evidence of a dominant dark matter component, but as direct evidence of the failure of the current Newtonian and general relativistic theories of gravity, in the large-scale or low-acceleration regimes relevant to the above. Some recent examples of the above are Milgrom \& Sanders (2003), Sanders \& Noordermeer (2007), Nipoti et al. (2007), Famaey et al. (2007), Gentile et al. 2007), Tiret et al. (2007) and Sanchez-Salcedo et al. (2008).

The range of galactic dynamical problems treated with the MOND formalism has extended from the first-order gravitational effects of rotation curves and velocity dispersion measurements to cover a wide range of more subtle problems. With their role in limiting the sizes of satellites and establishing in MOND escape velocities for satellites or galaxies subject to an external acceleration field, tidal forces were studied by Sanchez-Salcedo \& Hernandez (2007). Wu et al. (2008) calculated the escape velocity for the Milky Way using both MOND and dark matter, and conclude that the LMC appears as bound from both points of view, in spite of the recently determined high proper motion for this object, with slightly better fits using the MOND prescription. Sanchez-Salcedo et al. (2008) looked at the thickness of the extended HI disk of the Milky Way from both angles and find a somewhat better fit to observations in the MOND theory. Sanchez-Salcedo et al. (2006) and Nipoti et al. (2008) examined the problem of dynamical friction in dSph galaxies, comparatively assuming Newtonian gravity or MOND, with decay timescales for globular clusters being somewhat shorter in MOND. This is a potential problem, unless one allows large initial orbital radii for the observed globular clusters beyond the current extent of the stellar populations.

Going to cosmological scales, Skordis et al. (2006) have studied the cosmic microwave background, Halle et al. (2008) looked at the problem of the cosmic growth of structure, Zhao et al. (2006) studied gravitational lensing of galaxies, and Angus et al. (2007) and Milgrom \& Sanders (2008) studied dynamics of clusters of galaxies. All of the above find the MOND description of the problem to be a viable option within the observational errors of the relevant determinations, despite the need to include some unseen mass on galaxy cluster scales. Options for the above include the cluster baryonic dark matter proposed by 
Milgrom (2008b), or the inclusion of neutrinos, whether active as studied by Sanders (2003) and Angus et al. (2007) or sterile as proposed by Angus et al. (2010), always within plausible expectations. Also, in spiral galaxies, although within observational errors, rotation curves have been reported by Gentile et al. (2007) and Corbelli \& Salucci (2007) to be somewhat less wellfitted assuming the MOND prescription than when using cored dark matter haloes.

Observationally, it appears that MOND fares as well as dark matter in accounting for measured dynamics, in all but the smallest scales, the case of local dSph galaxies remains the most controversial. Published studies using MOND, which typically assume the deep MOND regime, sometimes find a different value for the acceleration scale of the theory for different $\mathrm{dSph}$ galaxies, when fitting detailed dynamical models to the data (Lokas 2001), or that $M / L$ ratios remain higher than those of stellar populations, making dark matter necessary even under the MOND formulation, e.g., Sanchez-Salcedo \& Hernandez (2007). Still, this last point remains somewhat controversial. Angus (2008) suggests that the MOND proposal will hold for local dSph galaxies, once the effects of tidal disruption are adequately incorporated.

Regarding corresponding theoretical developments, numerous alternative theories of gravity have recently appeared (Bekenstein 2004; Sanders 2005; Sobouti 2007; Mendoza \& Rosas-Guevara 2007; Bruneton \& Esposito-Farese 2007; Zhao 2007; Arbey 2008), now mostly grounded on geometrical extensions of general relativity and field theory, which lead to laws of gravity in the Newtonian limit that in the large-scale or lowacceleration regime reduce to the MOND prescription fitting formula.

The MOND theory is characterised by: (i) a low acceleration regime where dynamics mimic the presence of dark matter; (ii) a high acceleration regime where Newtonian gravity is recovered; and (iii) a somewhat ill-defined transition region. Variants of MOND are defined by the form of the interpolation function, $\mu(x)$, which mediates the transition between the two limit regimes, see Eq. (1).

We show how the MOND prescription with a suitable interpolation function, such as the one used by Bekenstein (2004) and used by Famaey \& Binney (2005), can be written as the addition of both low acceleration limit MOND and Newtonian contributions to the acceleration on all scales. The large differences in scales and magnitudes of the two acceleration terms ensure that the addition does not spoil the good match with observations on galactic scales. We are trying to look for a suitable MOND interpolation function for the local $\mathrm{dSph}$ galaxies, but not necessarily one that works on all scales.

This, as it is the regime of the local dSph galaxies, the one which has proven most difficult for MOND, and the one where the high-quality recent observations now available allow for a relatively clean test. We find that this interpolation function not only yields acceptable $M / L$ values for all dSph galaxies without the need for any dark matter in all cases, but also provides a natural explanation for all the scalings seen in these Galactic satellites. An interesting correlation between the $M / L$ ratios inferred and the ages of the stellar populations in local dSph galaxies is found, and naturally accounted for in modified gravity schemes where stars alone account for the gravitational force.

However, on solar system scales, Sanders (2006) has shown this particular interpolation function to be incompatible with limits on the variations of Kepler's constant. Thus, the good accordance we find with observations for dSph's must be understood as evidence in favour of the particular interpolation function we test, at the weak field limit relevant for these systems. What a full interpolation function for MOND should be is a more complicated issue. One, however, which has to be addressed bearing in mind the results presented here.

Section 2 gives a brief summary of the interpolation function first explored by Bekenstein (2004) and gives equilibrium isothermal configurations. These are then used in Sect. 3 to model the local $\mathrm{dSph}$ galaxies and obtain $M / L$ ratios, which are found to be consistent with those typical of stellar populations, at the ages of the different dSph systems. Section 4 includes an exploration of the most conspicuous scaling relations for local dSph galaxies, finding that Bekenstein's interpolation function naturally accounts for all features found. Our conclusions are summarised in Sect. 5.

\section{A particular form of the interpolating function in the MOND prescription}

In terms of the acceleration $\boldsymbol{g}$ felt by a test particle, the MOND proposal is (see e.g. Milgrom 2002)

$\mu\left(\frac{g}{a_{0}}\right) \boldsymbol{g}=\boldsymbol{g}_{\mathrm{N}}$

where $\boldsymbol{g}_{\mathrm{N}}$ is the acceleration assigned by Newtonian gravity, $a_{0}$ an acceleration scale of the theory, and the interpolating function $\mu\left(x:=g / a_{0}\right)$ is an unspecified function that reduces to unity for large values of its argument, and to its argument, for small values of it. In this way, Newtonian gravity is recovered for large values of $g$, and in the deep MOND regime one obtains

$g=\left(a_{0} g_{\mathrm{N}}\right)^{1 / 2}$.

Strictly speaking, MOND is a theory defined only at the limit values for the acceleration. The value of the constant $a_{0}$ has been reasonably determined by calibrating the deep MOND regime through observations of rotation velocity curves of large spiral galaxies (e.g. Sanders \& McGaugh 2002). However, the details of the transition region between the two regimes (i.e. the function $\mu$ ) have proven harder to establish reliably. This is in part natural, as it is not a scalar parameter, but a functional dependence that one is looking for here. A number of variants for this function have been proposed (e.g. Famaey \& Binney 2005; Zhao 2007; Sanders \& Noordermeer 2007), but apparently suitable dynamical systems in the transition region are hard to come by. Astrophysically, to first approximation one tends to find either systems where no dark matter is needed (stellar and planetary systems, globular clusters, vertical dynamics of disk galaxies, galactic bulges, and elliptical galaxies), or systems where dark matter is massively dominant ( $\mathrm{dSph}$ Galactic satellites, rotation curves of spiral galaxies, galaxy groups and clusters, and cosmological observations). Also, the details of any empirically inferred transition region, $\mu(x)$, are sensitive to the details of the baryonic system one is looking at, such as the assumed mass-to-light ratios, gas dynamics, or orbital anisotropy of stars, most of which play a marginal role when in the deep MOND regime. As pointed out already, this ill-defined transition is cumbersome to handle in attempts to reproduce the MOND phenomenology through simple GR extensions e.g. Sobouti (2007) or Capozziello et al. (2007).

Bekenstein (2004) shows that his relativistic extension of $\mathrm{TeVeS}$ in the appropriate non-relativistic limits yields the interpolating function

$\mu(x)=\frac{\sqrt{1+4 x}-1}{\sqrt{1+4 x}+1}$. 
This particular interpolating function converges to the right limits as $x \longrightarrow 0, \infty$ and has a very peculiar property. Direct substitution of Eq. (3) into the absolute value of relation (1) yields

$g=g_{\mathrm{N}}+\left(a_{0} g_{\mathrm{N}}\right)^{1 / 2}$.

This equation can be thought of as a generalised gravity recipe described by the addition of two terms, the first a standard Newtonian acceleration term and the second the MOND limit acceleration term. Seen in this way, Eq. (4) changes its acceleration behaviour limiting cases to a scale limiting behaviour. Indeed, for the case of a test particle on a gravitational field produced by a central mass $M$, located at a distance distance $R$ from it, Eq. (4) can be written as

$g=-\frac{G M}{R^{2}}-\frac{\left(G a_{0}\right)^{1 / 2} M^{1 / 2}}{R}$,

where $G$ is Newton's constant of gravity. Seen in this way, Eq. (5) converges to Newtonian gravity for sufficiently small $R / M^{1 / 2}$,s and reproduces the MOND strong limit for sufficiently large $R / M^{1 / 2}$ 's, and so the acceleration limits are now "scaleweighted" limits. It is worth noting that Eq. (5) is certainly the simplest modification to Newtonian gravity once the weak acceleration MOND regime is known.

Let us now rewrite Eq. (4) in such a way that the scale weighting becomes clearer. To do so, recall that $g_{\mathrm{N}}=-\nabla \phi_{\mathrm{N}}$, where $\phi_{\mathrm{N}}$ represents the standard Newtonian potential and $g=$ $-\nabla \phi$, where $\phi$ is the scalar potential of the gravitational field. With this, Eq. (4) can be written as

$g=g_{\mathrm{N}}(1+\chi)$,

where $\chi:=\left(a_{0} / G\right)^{1 / 2}\left(R / M^{1 / 2}\right)$. In what follows we use the standard values of $a_{0}=1 \times 10^{-8} \mathrm{~cm} \mathrm{~s}^{-2}$ (Milgrom 2008a) and $G=4.5 \times 10^{-39} M_{\odot}^{-1} \mathrm{~s}^{-2} \mathrm{kpc}^{3}$, in units suitable for galactic applications.

As already mentioned, we are interested in the form of $\mu(x)$ at scales of the local dSph galaxies. That the particular $\mu(x)$ we are testing is incompatible with solar system dynamics implies that a complete $\mu(x)$ MOND function is probably complex, that is, if one wants to treat the MOND formalism as more than just an empirical phenomenological description of gravitational physics on galactic scales. On the other hand, some researchers (see e.g. Bekenstein 2006 and references therein) claim that the Pioneer anomaly can be explained by the MOND hypothesis. This assumption can prove wrong if uneven thermal radiation in the spacecrafts is found (Toth \& Turyshev 2009), which could possibly come from the flyby anomaly (Turyshev 2009). In other words, the MOND formalism does not appear to be relevant on solar system scales, where General Relativity and Newtonian Gravity have proven correct (e.g. Turyshev \& Toth 2009; Anderson et al. 2002). Still, as discussed by Milgrom (2009), it is possible that current tests on solar system scales cannot reach definitive conclusions on the MOND interpolating function.

For globular clusters, with $M=10^{5-6} M_{\odot}, R=2-10 \times$ $10^{-3} \mathrm{kpc}$, we get values for $\chi$ of between 0.1 and 0.01 , and the correction becomes smaller than the errors and uncertainties in the observational determinations for the values of radii and masses for globular clusters. For elliptical galaxies and bulges, with masses going from about $10^{9}$ to $10^{11} M_{\odot}$ and radii of between 0.5 and $10 \mathrm{kpc}, \chi$ is about 0.1 . We thus see that the correction to gravitational dynamics due to the proposed inclusion of a second term in Eq. (6) is small enough to have remained undetected in galactic systems where no gravitational anomaly is found and where dynamics are consistent with Newtonian gravity, in the absence of any dark matter.

On the other hand, for systems where the presence of dark matter is inferred, given that this is always required to be dominant, adding the Newtonian term onto the MOND proposal generally provides a negligible contribution. For example, for the Galactic disk at the solar radius, $M=5 \times 10^{10} M_{\odot}$ and $R=$ $8.5 \mathrm{kpc}$ give $\chi=2$, consistent with an inference of about $50 \%$ dark matter within the solar circle. In going to the outskirts of the Milk Way, we go to $R=100 \mathrm{kpc}$, hence $\chi=20$. The system is either totally dominated by dark matter, or it is in a regime where the second term in Eq. (6) almost fully determines the dynamics. We see that the distinct power-law dependences ensure that the Newtonian term completely dominates at small values of $\chi$, while the opposite holds for large values of $\chi$, with a necessarily narrow transition region.

From the form of Eq. (5), it would be tempting to add the following term of the $\sim 1 / R$ series, a further (perhaps positive) constant term, which would result in an $\chi^{2}$ additive term in Eq. (6). If chosen suitably small, for the same reasons as given above, it would have no measurable effects on all but the largest scales, perhaps as a tool to model the cosmological constant. This extension certainly does not correspond to MOND, since no interpolation function $\mu(x)$ can be constructed. However, in such terms, Eq. (6) might be interpreted as a series expansion of a more fundamental underlying gravity law, only the first terms of which we have begun to appreciate empirically as observations probe increasingly higher $\chi$ regimes, typically corresponding to increasingly larger scales. The introduction of the constant term in Eq. (5) and its calibration from cosmology, however, must be done within a generalised GR framework.

\subsection{Equilibrium configurations}

To test at the regime which has resulted most troublesome for the MOND formalism, we go to the now very well studied dSph galaxies of the local group. To compare against local dSph galaxies, we require the derivation of equilibrium configurations for a population of self-gravitating stars. We begin by noting that the validity of Newton's theorems for spherically symmetric matter distributions, that the contribution to the force felt by an observer due to external shells vanishes, and that the contribution of all shells interior to the observer is equivalent to concentrating all mass interior to the observer at the centre, also holds under the present proposal. The two theorems depend on the numerator of the Newtonian term in Eq. (5) for a fixed solid angle fraction of a thin shell scaling with the second power of distance to the shell, as the denominator does. For the second term in Eq. (5), the numerator scales with $\left.\sqrt{(} R^{2}\right)=R$, as does the denominator, assuring the validity of Newton's theorems for spherical mass distributions (e.g. see Bekenstein \& Milgrom 1984).

We now write the equation of hydrostatic equilibrium for a polytropic equation of state $p=K \rho^{\gamma}$ :

$K \gamma \rho^{\gamma-2} \frac{\mathrm{d} \rho}{\mathrm{d} r}=-\nabla \phi=-\frac{G M(r)}{r^{2}}-\frac{\left(a_{0} / G\right)^{1 / 2} M(r)^{1 / 2}}{r}$

Since $\rho=\left(4 \pi r^{2}\right)^{-1} \mathrm{~d} M(r) / \mathrm{d} r$, where $M(r)$ is the mass of the configuration at a radius $r$, and going to isothermal conditions, $K=\sigma^{2}$ with $\gamma=1$, the previous equation can be written as

$\sigma^{2}\left[\left(\frac{\mathrm{d} M}{\mathrm{~d} r}\right)^{-1} \frac{\mathrm{d}^{2} M}{\mathrm{~d} r^{2}}-\frac{2}{r}\right]=-\frac{G M(r)}{r^{2}}-\frac{\left(a_{0} / G\right)^{1 / 2} M(r)^{1 / 2}}{r}$ 


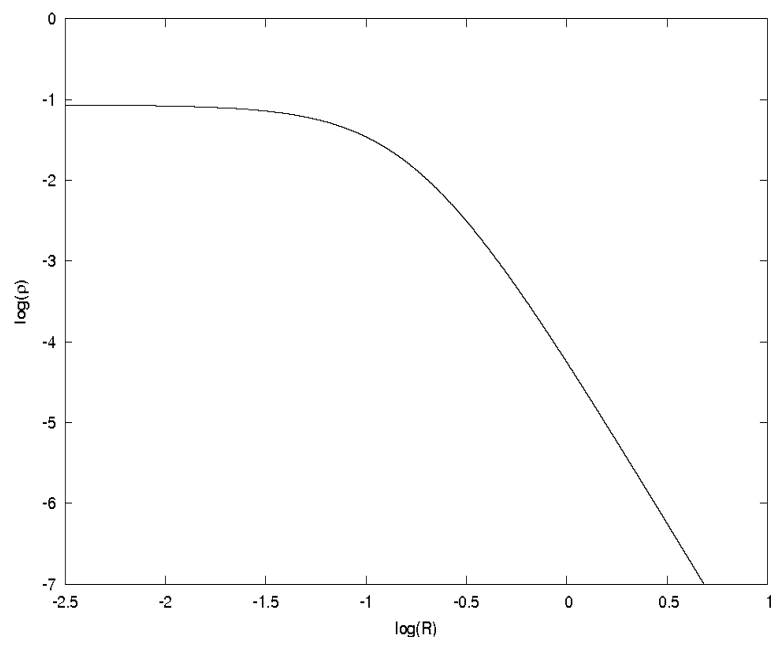

Fig. 1. A sample isothermal equilibrium density profile for $\rho_{0}=$ $0.1 M_{\odot} \mathrm{pc}^{-3}$ and $\sigma=7 \mathrm{~km} \mathrm{~s}^{-1}$, resulting in a total mass of $3 \times 10^{6} M_{\odot}$ and a volume half-mass radius of $0.39 \mathrm{kpc}$. The density is measured in units of $M_{\odot} \mathrm{pc}^{-3}$ and the distance in units of kpc.

In the above second-order differential equation for $M(r)$, the constant isotropic velocity dispersion for the population of stars has been written as $\sigma$. We have taken advantage of the correspondence between hydrostatic equilibrium polytropic configurations and self gravitating stellar systems, which does not depend on the particular form of the Poisson equation, only on the validity of the isothermal condition for the stellar population (see e.g. Binney \& Tremaine 2008). Taking initial conditions $M(r) \rightarrow 0$ and $\mathrm{d} M / \mathrm{d} r=4 \pi r^{2} \rho_{0}$ for $r \rightarrow 0$, a constant central density $\rho_{0}$, we can now solve Eq. (8) through a numerical finite differences scheme, subject only to two input conditions, a value for $\sigma$ and a value for $\rho_{0}$.

Notice that neglecting the first term on the right hand side of Eq. (8), the limit behaviour for $M(r)$ for large values of $r$ when the weak limit of the MOND formalism dominates, is characterised by a finite total mass. In contrast to what happens in Newtonian systems where isothermal configurations have an infinite total mass, isothermal self-gravitating configurations will be naturally bound in mass, under the proposed gravity law. As a result, there will also be a well-defined and finite half-mass radius, $R_{\mathrm{hm}}$, to characterise the resulting equilibrium configurations. Also, as is the case using the MOND prescription, the total mass of the configuration is expected to scale with $\sigma^{4}$. From the dimensional mass scale $\langle M\rangle \sim \sigma^{4}\left(a_{0} / G\right)^{-1}$, one is led to expect an analogue to the Tully-Fisher relation for spheroidal galactic systems, down to the $\mathrm{dSph}$ regime.

Figure 1 gives a plot of $\log \left(\rho / M_{\odot} \mathrm{pc}^{-3}\right)$ vs $\log (R / \mathrm{kpc})$ for a sample numerical solution to Eq. (8) for $\sigma=7 \mathrm{~km} \mathrm{~s}^{-1}$ and $\rho_{0}=$ $0.1 M_{\odot} \mathrm{pc}^{-3}$. We obtain a well-defined resulting total mass, $M_{\mathrm{tot}}$, of $3 \times 10^{6} M_{\odot}$, and a final volume half-mass radius of $0.39 \mathrm{kpc}$. We see the expected decay of the density profile at large radii, slightly faster than $r^{-3}$. It is this type of density profiles that is used in the next section to model the local dSph galaxies.

\section{Local dwarf spheroidal galaxies}

As discussed above, once values for the velocity dispersion and the central density are given, numerically solving Eq. (8) yields the full equilibrium density profile. To now model $\mathrm{dSph}$ galaxies, we take values for the reported velocity dispersions for these systems, which thus fix one of the two parameters of the model.
A resulting density profile is then integrated along one dimension to yield a surface density profile, from which the projected half-mass radius is measured. This provides a second constraint; we then vary the value of the input central density to ensure that the resulting half-mass radius $R_{\mathrm{hm}}$ matches the reported halflight radius $R_{\mathrm{hl}}$ of a given dSph. Observational determinations of the velocity dispersion and the half-light radius of a certain $\mathrm{dSph}$ galaxy then fully determine the model. Dividing the total mass for the resulting model $M_{\text {tot }}$ by the reported total luminosity $L_{\text {tot }}$ of a dSph, yields a mass to light ratio for the model. Values of the half-light radii and total luminosities were taken from Gilmore et al. (2007), who summarise published results from the references given in Table 1, except for Ursa Minor, where we took the revised value for $L_{\text {tot }}$ from Palma et al. (2003).

Although other stellar systems show velocity dispersion profiles that generally decay as the radial coordinate increases, e.g. globular clusters, the case for observed dSph galaxies is different, with these systems showing essentially flat velocity dispersion profiles, e.g. Gilmore et al. (2007). In cases where some drop in the velocity dispersion profile is observed, this typically occurs towards the edge of the galaxy, affecting only a very small percentage of the total mass of the system. The constancy of these observed velocity dispersion profiles validates the use of Eq. (8) under isothermal conditions for modelling local dSph's. We have not at this point attempted a more detailed modelling considering non-isothermal conditions, for example including orbital anisotropy, although such models are now common in models of dSph galaxies under Newtonian (e.g. Lokas 2002) or MOND frameworks (e.g. Angus 2008) and could be included in subsequent analysis. We point to the recent work by Gilmore (2007), in which isothermal conditions are used to model local dSph galaxies assuming Newtonian gravity.

Values of $\sigma$ were taken from adjusting a constant level to the $\sigma$ profiles of Angus (2008), which are flat to a very good approximation, with the exception of Ursa Minor that shows a significant decrease with increasing radial distances. This galaxy also shows internal structure in phase space at small radii, which might partly account for the steep increase in $\sigma$ seen by Angus (2008) towards the centre. We have taken a value for $\sigma$ representative of the situation at around the half-light radius. If a strong radial dependence of $\sigma$ in Ursa Minor were confirmed, however, this system would have to be excluded from the present sample, as it would then conflict with the simple isothermal modelling we are performing here.

Table 1 gives our results for the 8 best-studied local dSph galaxies. The values we obtain for $M / L$ are mostly comparable to those obtained by Angus (2008) for the same systems, but a slight systematic decrease is evident, consistent with having included a further force term, the Newtonian component, which reduced inferred $M / L$ ratios even further. In general, the values of the parameter $\langle\chi\rangle$ we found, calculated from the resulting model total mass as $\left(a_{0} / G\right)^{1 / 2}\left(2^{1 / 2} R_{\mathrm{hl}} / M_{\text {tot }}^{1 / 2}\right)$, explain why the addition of the Newtonian term is required to fully account for the dynamics. Further, the wide range of values of $\langle\chi\rangle$ shown in the table explains why a different $a_{0}$ has sometimes been found for various dSph's, when fitting dynamics using only the weak acceleration limit of the MOND formalism, e.g. Lokas (2001).

The case where the effect of the second term in Eq. (6) is greatest is that of Draco, where the compact configuration and relatively high $\sigma$ values allow the Newtonian term to figure somewhat more, leading to a strong decrease in the inferred $M / L$ of between 8.1 and 30.9, compared to the Angus (2008) results of $M / L$ between 22.6 and 72.9. With the possible exception of Fornax and Sculptor, cases where the reported King core 
X. Hernandez et al.: Dwarf spheroidals in MOND

Table 1. Basic properties and resulting $M / L$ ratios for the sample of dSph galaxies.

\begin{tabular}{llllllll}
\hline \hline Galaxy & $\sigma\left[\mathrm{km} \mathrm{s}^{-1}\right]$ & $R_{\mathrm{hl}}[\mathrm{kpc}]$ & $L_{\mathrm{tot}} \times 10^{5} L_{\odot}$ & $(M / L)_{A}$ & $(M / L)$ & $\langle\chi\rangle$ & $\begin{array}{l}\text { Age of Youngest } \\
\text { Component [Gyr] }\end{array}$ \\
\hline Carina & $7 \pm 1.8$ & 0.290 & 4.3 & $5.6_{-2.9}^{+5.2}$ & $6.8_{-4.6}^{+8.3}$ & 7 & 3 \\
Draco & $8 \pm 1.5$ & 0.230 & 2.6 & $43.9_{-19.3}^{+29}$ & $17.0_{-8.9}^{+13.9}$ & 4.1 & 10 \\
LeoI & $8 \pm 1.2$ & 0.330 & 48.0 & $0.7_{-0.3}^{0.65}$ & $1.0_{-0.44}^{+0.6}$ & 5.7 & 2 \\
Sextans & $7 \pm 1.0$ & 0.630 & 5.0 & $9.2_{-3.0}^{+5.3}$ & $6.3_{-2.8}^{+4.2}$ & 13.4 & $(2-6)$ \\
Fornax & $12 \pm 1.3$ & 0.400 & 150.0 & $1.4_{-0.35}^{+0.45}$ & $1.4_{-0.4}^{+0.6}$ & $>3.4$ & $(2-3)$ \\
Sculptor & $9.5 \pm 1.7$ & 0.160 & 22.0 & $3.7_{-1.4}^{+2.2}$ & $3.4_{-0.7}^{+2.1}$ & $>2.2$ & $>5$ \\
LeoII & $6 \pm 1.4$ & 0.185 & 7.0 & $1.85_{-1.1}^{+2}$ & $2.3_{-04}^{+2.4}$ & 5.5 & 6.5 \\
Ursa Minor & $8 \pm 2$ & 0.300 & 5.8 & $5.8_{-3.6}^{+6.5}$ & $8.0_{-5.1}^{+9.8}$ & $>5.3$ & 12 \\
\hline
\end{tabular}

Notes. $(M / L)_{A}$ gives the values for the mass to light ratios calculated by Angus (2008), and $M / L$ those in this study under the proposed model. Total luminosities (in the $V$ band) and half-light radii are from Wilkinson et al. (2006), Wilkinson et al. (2004), Koch et al. (2007a), Kleyna et al. (2004), Walker et al. (2006), Mateo (1998), Coleman et al. (2007) and Irwin \& Hatzidimitriou (1995), for the galaxies in the table, in the order given, as summarised in Gilmore et al. (2007). Velocity dispersions are from adjusting a constant value to the data of Angus (2008). $\langle\chi\rangle$ gives the average value of the parameter $\chi$ as defined in Sect. 2, which measures the relative relevance of the Newtonian and the low acceleration limit of the MOND formalism terms in Eq. (6). The final column gives an estimate of the age of the youngest stellar population present in each of the systems, for Carina, Ursa Minor, LeoI, and LeoII from Hernandez et al. (2000), for Fornax from Coleman \& de Jong (2008), for Draco from Aparicio et al. (2001), for Sextans from Lee et al. (2003), and for Sculptor from Babusiaux et al. (2005).

radius serves only as a lower limit for the projected $R_{\mathrm{hl}}$, the value for the parameter $\langle\chi\rangle$ for Draco is the lowest in the sample. This naturally explains why it is here that our results for $M / L$ differ most and show a significant reduction when contrasting with the results of Angus (2008), who takes a $\mu$ function that rapidly converges to the low acceleration limit of the MOND prescription, as this is the parameter that determines the relative importance of the Newtonian and low acceleration limit MOND terms in Eq. (6).

The $1 \sigma$ confidence intervals of our results are only lower bounds, as their calculation includes only uncertainties in the adopted values of $\sigma$ and not observational errors in total luminosities and half-mass radii. Also, freedom in the light profile functional fitting would increase our confidence intervals slightly, e.g., the reported $R_{\mathrm{hl}}$ values for Ursa Minor, Fornax and Sculptor are only lower bounds, because the quantity available for those galaxies is the core radius from King profile fits. Still, this last is only a second-order effect, since our inferred $M / L$ values are not very sensitive to the values of $R_{\mathrm{hl}}$ used, provided this does not change by a large factor (see the following section).

As expected, the main determinant of the resulting total mass of a numerical solution of Eq. (8), is the input $\sigma$. We find, as expected from the situation of the low acceleration limit of the MOND prescription, a strong correlation, with the resulting scaling for all galaxies being well described by

$M_{\mathrm{tot}}=\frac{\left(3.36 \sigma^{2}\right)^{2}}{a_{0} G}$.

This could be seen as an extension of the Tully-Fisher relation down to the smallest galactic scales, a natural consequence of the model being explored.

Considering the large $M / L$ values natural for old stellar populations, e.g., Queloz et al. (1995) and Romanowsky (2003), who find $M / L$ ratios for old stellar populations as high as 7 or 8 , our new results are now at the limit of compatibility with $M / L$ ratios for old naked stellar populations for all the well-studied local dSph galaxies. This holds comfortably for all galaxies except Draco, where compatibility is found only just at the $1 \sigma$ level for our lower limits on the confidence intervals. Our model thus yields equilibrium isothermal configurations able to explain the observed dynamics of the local dSph galaxies, without the need for dark matter.

A consistency check of the above interpretation is available from the comparison of the $M / L$ ratios we found and the age of the youngest stellar population found in each of the galaxies, as inferred from the direct studies of the observed CMDs of the galaxies in question, given in the last column of Table 1. It is reassuring that the highest values for our inferred $M / L$ ratios, for Ursa Minor and Draco coincide with the galaxies showing no star formation over the past $10 \mathrm{Gyr}$, the oldest galaxies in the sample, while the lowest values for $M / L$ are obtained for Leo I and Fornax, the youngest galaxies in the sample with stellar populations as young as 2 Gyr. For the remainder of the sample, galaxies showing their youngest stars at intermediate ages, we find the intermediate values for $M / L$. The interpretation of the dynamics under the proposed model thus accords with the natural increase in the $M / L$ ratios of stellar populations due to the ageing of stars and the consequent build up of black holes, neutron stars, and white dwarfs. This correspondence is natural in any modified gravity scheme where the stars alone are responsible for the dynamics, but has to be thought of as a fortuitous coincidence under the dark matter hypothesis.

As a second consistency check, we now show the resulting projected surface density mass profile for a model of the Leo II galaxy, normalised by the total luminosity of that system. We construct the surface brightness profile using our model for an equilibrium isothermal system having velocity dispersion and projected $R_{\mathrm{hm}}$ (assumed equal to the observed $R_{\mathrm{hl}}$ ), equal to those observed for Leo II. This is given in Fig. 2, where we have also plotted the observation for the actual surface density light profile 


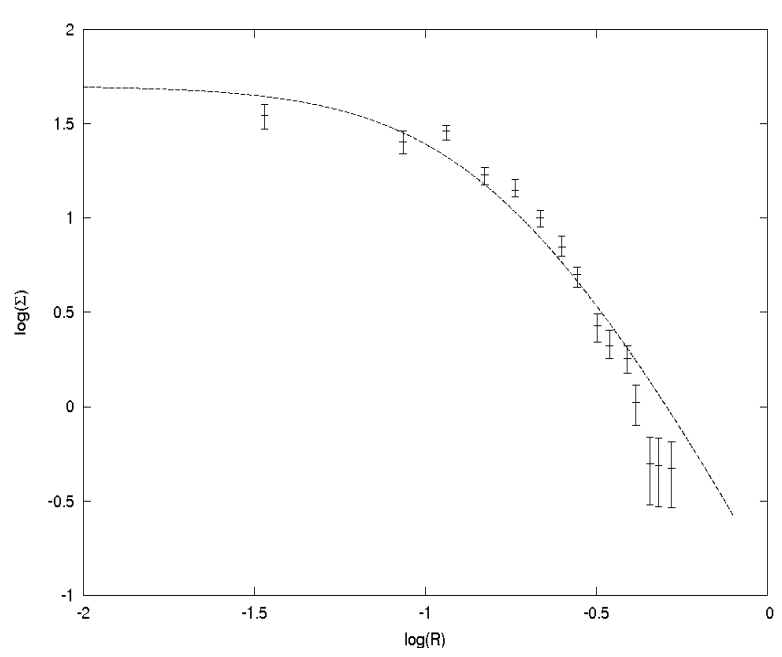

Fig. 2. Comparison of our projected surface density profile for an equilibrium isothermal solution to Eq. (8), having input $\sigma$ and projected $R_{\mathrm{hm}}$ as the observationally determined values for LeoII, dashed curve. For comparison, we also give the star counts surface density profile for Leo II of Coleman (2007). Both profiles have been normalised to the same total luminosity.

of Leo II, from the star counts analysis of Coleman et al. (2007), out to the radius where measurements fall below the background noise level, both normalised to the same total luminosity. We note that the error bars of Coleman et al. (2007) are only a lower estimate of the confidence intervals for this comparison, as they only refer to the errors in the measured star counts and not to full surface density profile inferences. A very good agreement is evident, showing the proposed models are a good, fully selfconsistent representation of the dynamics and both the integral and spatially resolved light distribution in the well-studied local dSph galaxies.

\section{Scaling relations}

We now turn to the scalings shown by the dSph galaxies in our sample. Firstly, we show in Fig. 3 the behaviour of the equilibrium isothermal configurations we are solving for, in terms of the resulting projected $R_{\mathrm{hm}}$ as a function of the input value of $\rho_{0}$, at a constant value of $\sigma=10 \mathrm{~km} \mathrm{~s}^{-1}$. The remarkable feature of Fig. 3 is that, after decreasing as $\rho_{0}$ increases, when $\rho_{0}$ reaches a value of about $0.3 M_{\odot} \mathrm{pc}^{-3}$, the resulting $R_{\mathrm{hm}}$ stops changing and settles at relatively constant value of around $150 \mathrm{pc}$, below which it does not fall further. This is qualitatively reproduced at all values of $\sigma$, with only small changes in the minimum values of $R_{\mathrm{hm}}$, for $\sigma$ in the range of values observed for local dSph galaxies. This is interesting, as it offers a natural explanation for local $\mathrm{dSph}$ galaxies showing a minimum lower value for their projected half-light radii of around $150 \mathrm{pc}$, with most lying around a factor of 2 above this critical limit, as noticed by Gilmore et al. (2007) and seen from Table 1. Indeed, all our models constrained by the observational values of $\sigma$ and $R_{\mathrm{hl}}$ for the local dSphs, occur within the flat region of the $R_{\mathrm{hm}}$ vs. $\rho_{0}$ space.

We can now try to understand the assigned Newtonian values of $M / L$ for local dSph galaxies and the scalings they show with total luminosity or absolute magnitude, e.g., Mateo (1998). The assigned Newtonian values of $M / L$ will never be far from (e.g. Gilmore et al. 2007)

$(M / L)_{\mathrm{N}}=\left(\frac{10 \sigma^{2} R_{\mathrm{hl}}}{G}\right)\left(\frac{1}{L_{\mathrm{tot}}}\right)$.

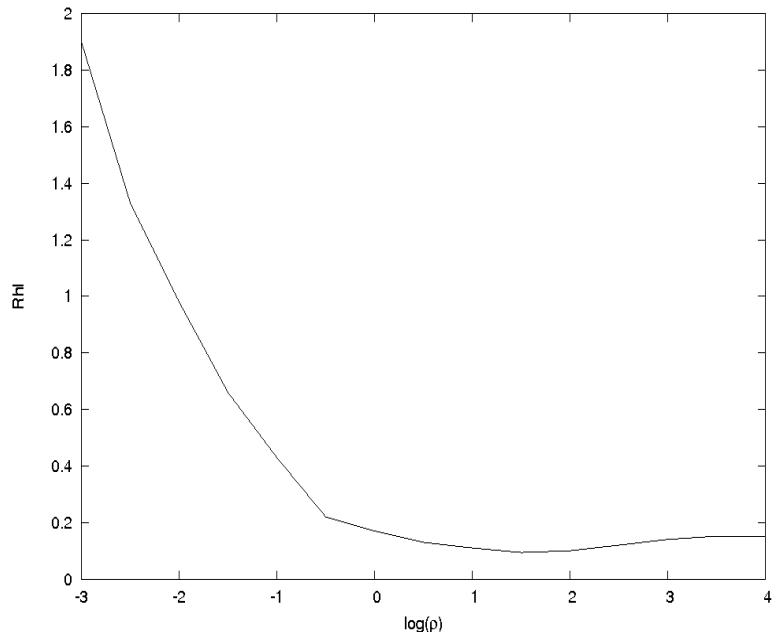

Fig. 3. Dependence of the resulting projected half-mass radius in kpc, against the assumed central density in $M_{\odot} \mathrm{pc}^{-3}$, for isothermal equilibrium configurations under the proposed MOND formalism, at fixed $\sigma=10 \mathrm{~km} \mathrm{~s}^{-1}$. A very broad region where $R_{\mathrm{hm}}$ remains almost constant is evident, at precisely the level found to define the minimum $R_{\mathrm{hl}}$ values for observed dSph galaxies, which have typical values of $\sigma \simeq 10 \mathrm{~km} \mathrm{~s}^{-1}$.

Given our results in the previous section, or alternatively taking the observed $R_{\mathrm{hl}}$ of around $300 \mathrm{pc}$ as an empirical fact, we can evaluate the above equation to first order at $R_{\mathrm{hl}}=0.3$. Also, for an average old stellar population, we can take a constant $M / L$ value of 5 as a representative value for the local dSphs, as our inferences yield, and replace $\sigma^{2}$ in Eq. (9) for the corresponding value through Eq. (10) to yield

$$
(M / L)_{\mathrm{N}}=\left(\frac{3 \sqrt{5}}{3.36}\right)\left(\frac{a_{0}}{G}\right)^{1 / 2}\left(\frac{1}{L_{\mathrm{tot}}}\right)^{1 / 2}
$$

Introducing the absolute magnitude $M_{V}=-2.5 \log \left(L_{\text {tot }}\right)+4.83$ and taking the logarithm of the above equation gives

$$
\log (M / L)_{\mathrm{N}}=3.77+0.2 M_{V} \text {. }
$$

Figure 4 now gives a plot of Eq. (12), superimposed on recent determinations of the Newtonian $M / L$ values for a larger sample of local dSph galaxies, using reported values of $L$ to calculate the values plotted on the $x$-axis, and of $L, \sigma$, and $R_{\mathrm{hl}}$ to calculate Newtonian $M / L$ values through Eq. (10). Error bars give extreme $1 \sigma$ confidence intervals, including uncertainties in all the parameters used. Data as summarised in Walker et al. (2009b) (using the revised version of the data for the classical dSphs, as given in Walker et al. 2009d), and references therein. The good match is evident, extending for over 13 orders of magnitude in $M_{V}$, which is even more remarkable because the trend found extends towards the smallest newly discovered dSph systems.

We see that the expectations of the model for the inferred Newtonian $M / L$ values of local dSph galaxies, which are modelled as equilibrium isothermal solutions to Eq. (8) and having typical radii of close to $0.3 \mathrm{kpc}$, agree very well with independent measurements. The small scatter in Fig. 4 beyond observational error bars is compatible with the variations in the actual intrinsic $M / L$ values for the individual galaxies, and variations of $R_{\mathrm{hl}}$ around the average $0.3 \mathrm{kpc}$ used in Eq. (12), which is not a fit to the data, but a first-order estimate within our prescription. A very simple explanation for the $(M / L)_{\mathrm{N}}$ values assigned to all well-studied dSphs is thus naturally afforded by our assumptions for the most average intrinsic $M / L$ and $R_{\mathrm{hl}}$ values, without 


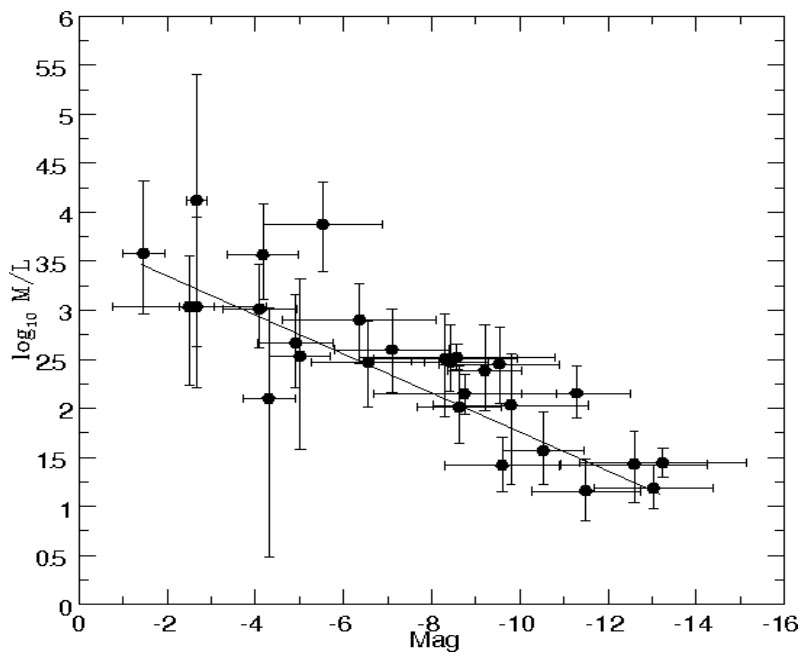

Fig. 4. Logarithms of the Newtonian $M / L$ values of local dSph galaxies against total observed $V$ band magnitudes, from data as summarised in Walker et al. (2009a). The straight line is not a fit, but gives the Newtonian $M / L$ values that would be assigned to isothermal equilibrium populations of stars having a constant intrinsic stellar $M / L$ ratio of 5 and a constant half-mass radius of $300 \mathrm{kpc}$, consistent with what is observed, and to what the proposed model yields for equilibrium configurations having velocity dispersions of $10 \mathrm{~km} \mathrm{~s}^{-1}$, as the observed dSph show, according to the model presented here. Galaxies in ascending order of luminosity, with original references: $1-\operatorname{Sgr}(23$, 24), 2- Fornax (1,2), 3- And II (17, 18), 4- Leo I (1, 5), 5- Cetus (17, 22), 6- Sculptor $(1,2), 7-$ And XV $(20,21), 8-$ Leo II $(1,6)$, 9- Tucana $(25,26), 10-$ Sextans $(1,2), 11-$ Draco $(3,4), 12-$ Carina $(1,2), 13-$ Cven I $(3,10), 14-$ Umi (1), 15- And IX (19), 16- Leo T (3, 10, 14), 17- Hercules $(3,11), 18$ - Bootes $1(3,8)$, 19- UMa I (3, 8), 20- Leo IV $(3,10), 21-$ Cven II $(3,10), 22$ - Leo V $(12,13)$, 23- UMa II $(3,10), 24-$ Coma $(3,10), 25-$ Willman $1(3,8), 26$ - Bootes $2(3,9), 27-$ Segue $2(16), 28$ - Segue $1(3,15)$. References: 1- Irwin \& Hatzidimitriou (1995), 2- Walker et al. (2009c), 3- Martin et al. (2008), 4- Walker et al. (2007), 5- Mateo et al. (2008), 6- Koch et al. (2007b), 7- Martin et al. (2007), 8- Koch et al. (2009), 9- Simon \& Geha (2007), 10- Aden et al. (2009), 11- Belokurov et al. (2008), 12- Walker et al. (2009b), 13- Irwin et al. (2007), 14- Geha et al. (2009), 15- Belokurov et al. (2009), 16- McConnachie \& Irwin (2006, 17- Cote et al. (1999), 18-Chapman et al. (2005), 19- Ibata et al. (2007), 20- Letarte et al. (2009), 21- Lewis et al. (2007), 22- Ibata \& Irwin (1997), 23- Majewski et al. (2003), 24- Saviane et al. (1996), 25- Fraternali et al. (2009).

the need of invoking complex astrophysical processes (e.g. tidal disruption, strong outflows, etc.) or fine-tuning any parameters. To first order, one can understand the well-established scalings of Fig. 4 as the extension of the Tully-Fisher relation, which appears in the MOND formalism, down to the $\mathrm{dSph}$ regime.

\section{Conclusions}

We used a MOND interpolation function that can be seen as the addition of both the Newtonian acceleration and the low acceleration limit of the MOND formalism on all scales (Bekenstein 2004). For the local dSph galaxies, where the application of the MOND formalism has been most controversial, we show that isothermal equilibrium configurations characterised by welldefined finite total masses and half-mass radii result, giving $M / L$ values in agreement with naked stellar populations. As a result, no dark matter is now needed. The observed scalings in $R_{\mathrm{hl}}$ and assigned $(M / L)_{\mathrm{N}}$ values as a function of total magnitudes are explained naturally, including the previously unrecognised correlation of resulting $M / L$ ratios with the relative youth of the stellar populations of the individual $\mathrm{dSph}$ galaxies, for the sample studied. This last is a natural consequence of using the MOND prescription, but an odd coincidence under the dark matter hypothesis.

Fixing the optimal $\mu(x)$ for the MOND prescription in the $\mathrm{dSph}$ regime through the arguments presented here constitutes an interesting condition on this function in the regime studied, and it provides a constraint on any global $\mu(x)$ MOND function.

Acknowledgements. We would like to thank Benoit Famaey, Moti Milgrom, and Jacob Bekenstein for fruitful comments in connection to a previous version of this article. We thank an anonymous referee for comments that were useful towards reaching a more balanced and complete presentation. This work was supported in part through two DGAPA-UNAM grants (PAPIIT IN-1130073 and IN-114107). S.M. and T.B. gratefully acknowledge support from DGAPA (IN119203-3) at the Universidad Nacional Autónoma de México (UNAM). S.M. acknowledges financial support by CONACyT (26344). T.B. acknowledges support from CONACyT (207529).

\section{References}

Aden, D., Feltzing, S., Koch, A., et al., A\&A 2009, 506, 1147

Arbey, A. 2008, OAJ, 1, 27

Anderson, J. D., Lau, E. L., Turyshev, S., Williams, J. G., \& Nieto, M. M. 2002, in BAAS, 660, 34

Angus, G. W. 2008, MNRAS, 387, 1481

Angus, G. W., Shan, H. Y., Zhao, H. S., et al. 2007, ApJ, 654, L13

Angus, G. W., Famaey, B., \& Diaferio, A. 2010, MNRAS, 402, 395

Aparicio, A., Carrera, R., \& Martínez-Delgado, D. 2001, AJ, 122, 2524

Babusiaux, C., Gilmore, G., \& Irwin, M. 2005, MNRAS, 359, 985

Bekenstein, J. D. 2004, Phys. Rev. D, 70, 083509

Bekenstein, J. 2006, Contemporary Physics, 47, 387

Belokurov, V., Walker, M. G., \& Evans, N. W., et al. 2008, ApJ, 686, L83

Belokurov, V., Walker, M. G., Evans, N. W., et al. 2009, MNRAS, 397, 1748

Bruneton, J. P., \& Esposito-Farese, G. 2007, Phys. Rev. D, 76, 124012

Chapman, S. C., Ibata, R., Lewis, G. F., et al. 2005, ApJ, 632, L87

Coleman, M. G., Jordi, K., Rix, H.-W., Grebel, E. K., \& Koch, A. 2007, AJ, 134, 1938

Coleman, M. G., \& de Jong, J. T. A. 2008, ApJ, 685, 933

Corbelli, E., \& Salucci, P. 2007, MNRAS, 374, 1051

Côté, P., Mateo, M., Olszewski, E. W., et al. 1999, ApJ, 526, 147

Famaey, B., \& Binney, J. 2005, MNRAS, 363, 603

Famaey, B., Gentile, G., Bruneton, J.-P., et al. 2007, Phys. Rev. D, 75, 063002

Fraternali, F., Tolstoy, E., Irwin, M., et al. 2009, A\&A, 499, 121

Geha, M., Willman, B., Simon, J. D., et al. 2009, ApJ, 692, 1464

Gentile, G., Famaey, B., Combes, F., et al. 2007, A\&A, 472, L25

Gilmore, G., Wilkinson, M. I., Wyse, R. F. G., et al. 2007, ApJ, 663, 948

Halle, A., Zhao, H. S., \& Li, B. 2008, ApJS, 177, 1

Hernandez, X., Gilmore, G., \& Valls-Gabaud, D. 2000, MNRAS, 317, 831

Ibata, R. A., \& Irwin, M. J. 1997, AJ, 113, 1865

Ibata, R., Martin, N. F., Irwin, M., et al. 2007, ApJ, 671, 1591

Irwin, M., \& Hatzidimitriou, D. 1995, MNRAS, 277, 1354

Irwin, M. J., Belokurov, V., Evans, N. W., et al. 2007, ApJ, 656, L13

Kleyna, J. T., Wilkinson, M. I., Evans, N. W., et al. 2004, MNRAS, 354, L66

Koch, A., Wilkinson, M. I., Kleyna, J. T., et al. 2007a, ApJ, 657, 241

Koch, A., Kleyna, J. T., Wilkinson, M. I., et al. 2007b, AJ, 134, 566

Koch, A., Wilkinson, M. I., Kleyna, J. T., et al. 2009, ApJ, 690, 453

Letarte, B., Chapman, S. C., Collins, M., et al. 2009, MNRAS, 400, 1472

Lee, M. G., Park, H. S., Park, J.-H., et al. 2003, AJ, 126, 2840

Lewis, G. F., Ibata, R. A., Chapman, S. C., et al. 2007, MNRAS, 375, 1364

Łokas, E. L. 2001, MNRAS, 327, L21

Łokas, E. L. 2002, MNRAS, 333, 697

Majewski, S. R., Skruskie, M. F., Weinberg, M. D., et al. 2003, ApJ, 599, 1082

Martin, N. F., Ibata, R. A., Chapman, S. C., Irwin, M., \& Lewis, G. F. 2007, MNRAS, 380, 281

Martin, N. F., de Jong, J. T. A., \& Rix, H. W. 2008, ApJ, 684, 1075

Mateo, M. L. 1998, ARA\&A, 36, 435

Mateo, M., Olszewski, E. W., \& Walker, M. G. 2008, ApJ, 675, 201

McConnachie, A. W., \& Irwin, M. 2006, MNRAS, 365, 1263

Mendoza, S., \& Rosas-Guevara, Y. M. 2007, A\&A, 472, 367

Milgrom, M. 1983, ApJ, 270, 365

Milgrom, M., \& Sanders, R. H. 2003, ApJ, 599, L25

Milgrom, M., \& Sanders, R. H. 2008, ApJ, 678, 131

Milgrom, M. 2008a, unpublished [arXiv:0801.3133] 
A\&A 514, A101 (2010)

Milgrom, M. 2008b, New Astron. Rev., 51, 906

Milgrom, M. 2009, MNRAS, 1143

Nipoti, C., Londrillo, P., Zhao, H., et al. 2007, MNRAS, 379, 597

Nipoti, C., Ciotti, L., Binney, J., \& Londrillo, P. 2008, MNRAS, 386, 2194

Palma, C., Majewski, S. R., Siegel, M. H., et al. 2003, AJ, 125, 1352

Queloz, D., Dubath, P., \& Pasquini, L. 1995, A\&A, 300, 31

Romanowsky, A. J., Douglas, N. G., Arnaboldi, M., et al. 2003, Science, 301, 1696

Sánchez-Salcedo, F. J., \& Hernandez, X. 2007, ApJ, 667, 878

Sánchez-Salcedo, F. J., Reyes-Iturbide, J., \& Hernandez, X. 2006, MNRAS, 370, 1829

Sánchez-Salcedo, F. J., Saha, K., \& Narayan, C. A. 2008, MNRAS, 385, 1585

Sanders, R. H. 2003, MNRAS, 342, 901

Sanders, R. H. 2005, MNRAS, 363, 459

Sanders, R. H. 2006, MNRAS, 370, 1519

Sanders, R. H., \& McGaugh, S. S. 2002, ARA\&A, 40, 263

Sanders, R. H., \& Noordermeer, E. 2007, MNRAS, 379, 702

Saviane, I., Held, E. V., \& Piotto, G. 1996, A\&A, 315, 40

Simon, J. D., \& Geha, M. 2007, ApJ, 670, 313

Skordis, C., Mota, D. F., Ferreira, P. G., \& Boehm, C. 2006, Phys. Rev. Lett., 96, 1301
Sobouti, Y. 2007, A\&A, 464, 921

Tiret, O., Combes, F., Angus, G. W., et al. 2007, A\&A, 476, L1

Turyshev, S. G. 2009, Physics Uspekhi, 52, 1

Toth, V. T., \& Turyshev, S. G. 2009a, Phys. Rev. D, 79, 043011

Turyshev, S. G., \& Toth, V. T. 2009b, Space Sci. Rev., 86

Walker, M. G., Mateo, M., Olszewski, E. W., et al. 2006, AJ, 131, 2114

Walker, M. G., Mateo, M., Olszewski, E. W., et al. 2007, ApJ, 667, L53

Walker, M. G., Mateo, M., Olszewski, E. W., et al. 2009a, ApJ, 704, 1274

Walker, M. G., Belokurov, V., Evans, N. W., et al. 2009b, ApJ, 694, L144

Walker, M. G., Mateo, M., Olszewski, E. W., Sen, B., \& Woodroofe, M. 2009c, AJ, 137, 3109

Walker, M. G., Mateo, M., Olszewski, E. W., Peñarrubia, J., Evans, N. W., Gilmore, G. 2009d, ApJ, 704, 1274

Wilkinson, M. I., Kleyna, J. T., Evans, N. W., et al. 2004, ApJ, 611, L21

Wilkinson, M. I., Kleyna, J. T., Wyn Evans, N., et al. 2006, in EAS Publ. Ser. 20, ed. G. A. Mamon, F. Combes, C. Deffayet, \& B. Fort, 105

Wu, X., Famaey, B., Gentile, G., Perets, H., \& Zhao, H. S. 2008, MNRAS, 386, 2199

Zhao, H. S. 2007, ApJ, 671, L1

Zhao, H. S., Bacon, D. J., Taylor, A. N., \& Horne, K. 2006, MNRAS, 368, 171 